\title{
A Two Stage Optimal Strategy for Centralized Charging Stations Planning in High Voltage Distribution System
}

\author{
Ning Xiong ${ }^{1}$, Jie Wang ${ }^{1}$, Wenguang Zhu ${ }^{1}$, Shiyuan Zhong ${ }^{1}$, Min Wang ${ }^{1}$, and \\ Wei Tang ${ }^{1}$ \\ ${ }^{1}$ Jiangxi Electric Power Corporation Electric Power Economic Research Institute, City of Nanchang \\ Jiangxi 330043, China
}

Keywords: centralized charging station, two-stage dispatch, load shifting, wind power grid integration, automatic SOC regulation, fuzzy control strategy

Abstract. Charging and discharging control of centralized charging station becomes more important in power system operation. In order to achieve a load shifting and wind power stabilizing, this paper presents a two-stage optimal scheduling model for centralized charging stations based on the considerations of the users' requirements and battery limits. The first stage is a dispatch strategy in day ahead aimed at smoothing the composite curve of load and wind power, and a PSO algorithm with penalty function constraint is proposed to solve the model. The second stage established a 10 minutes real time scheduling policy of centralized charging station based on the results of the first stage optimization, in order to reduce the volatility of wind power curve through the automatic SOC regulating principle. Simulation results show that the two-stage dispatch can achieve load shifting and wind power stabilizing on the basis of meeting users' needs.

\section{Introduction}

Electric vehicles (EVs) can help to reduce carbon emission, however, it will also cause serious effects on power quality, reliability and node voltages [1-6]. Transfer capacities of distribution networks can be increased, as well as reliability and integrated capacities of distributed generations (DGs), because EVs can be used not only as loads, but also as distributed stored energy [7]. Therefore, EVs have been promoted and developed all over the world, and centralized charging strategies based on battery leasing have become a competitive business model [8-9]. A coordinated control between DGs and EVs is an important method in distribution networks, in order to mitigate power fluctuations.

Various methods have been proposed to research the coordinated relationship between EVs and power grids, which are mainly focused on battery changing models, and EVs stations are optimized as loads in order to achieve peak load shifting. Reference [10] proposed a day ahead active distribution network optimal schedule with EV penetration, in which the model is to take the lowest operating cost in each calculation period. Reference [11] considered scheduling EV charging load to improve the wind power penetration and reduce charging cost, in which EV load is formulated as the robust shortest path Markov Decision Process to obtain a robust charging policy. Reference [12] analyzed the electric vehicle (EV) charging scheduling problem to match the stochastic wind power. Besides considering optimization of the expected charging cost, the proposed model creatively incorporates the matching degree between wind power and EV charging load into the objective function.

This paper proposed a two-stage coordinated dispatching method, which can satisfy both battery changing requirement of customers and power balance requirement of DGs and loads. The limit of battery state of charge in $110 \mathrm{kV}$ centralized charging station is also considered in the proposed model. A day ahead dispatching strategy is determined in the first stage, in which a 24-hour scheduling of EVs station is optimized, aiming at balance power fluctuation of DGs and loads. The results of the first stage optimization are set as reference objectives in second stage optimization, in which a 10-minute real time scheduling is optimized in order to reduce power fluctuation in an hour. 


\section{Two-stage scheduling of centralized charging stations}

The studies of existing charging stations are often difficult to meet the various requirements at the same time. To achieve the two goals to meet the demand for electricity of users and control load and the fluctuation of wind power, we also consider the limitation of battery in state of charge. Therefore we put forward two-stage scheduling of centralized charging stations, and the overall framework is shown in Fig.1.

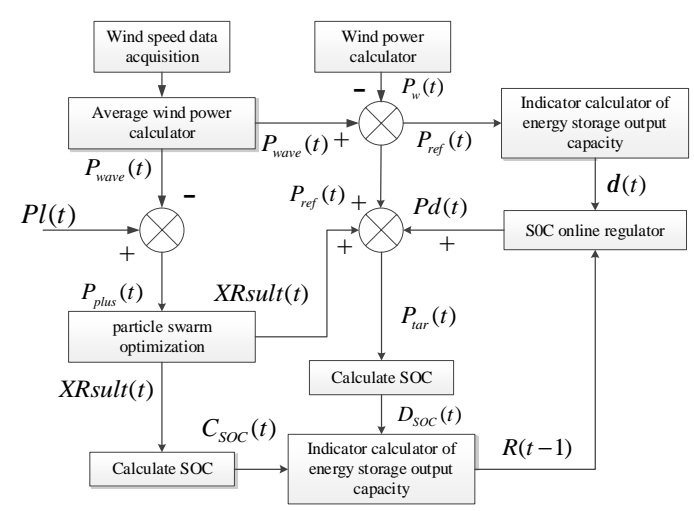

Fig.1 Framework of two-stage scheduling

In fig.1, the left side of the dotted line is day-ahead scheduling, the right side is Real-time Scheduling. Day-ahead Scheduling is to meet the needs of users as the limitation and achieve smoothing of equivalent curve; Real-time Scheduling achieves the overall scheduling through power correction. The scheduling result of Day-ahead Scheduling can be a reference value for Real-time Scheduling.

\section{The strategy of day-ahead scheduling}

The control objective of this stage is to meet the demand for electricity of EVs, and in this premise, the fluctuation of Wind equivalent power and load can be stabilized. Taking $1 \mathrm{~h}$ as the scheduling period, $1 \mathrm{~d}$ is divided into 24 periods.

\section{Objective function}

Day-ahead scheduling objective function is the minimum accumulated value of equivalent power fluctuation:

$$
\begin{gathered}
F=\min \sum_{t=2}^{24}\left(P_{E}(t)-P_{E}(t-1)\right)^{2} \\
P_{E}(t)=P_{l}(t)-P_{w a}(t)-P(t)
\end{gathered}
$$

where $P_{E}(t)$ is the equivalent load value at time t, whose unit is $\mathrm{MW} ; P_{l}(t)$ is the load value of distribution network at time $\mathrm{t}$, whose unit is $\mathrm{MW} ;{ }_{P_{w a}}(t)$ is the wind power value at time $\mathrm{t}$, whose unit is MW; $P(t)$ is the charge-discharge power value of centralized charging stations at time $\mathrm{t}$, whose unit is MW; $P(t)>0$ represents that centralized charging stations discharge to grid, and $P(t)<0$ represents that centralized charging station charge from grid.

\section{Constraint condition}

The constraint conditions of centralized charging stations to realize charging scheduling mainly shows in three aspects: the charging and discharging power of charging station, the battery power in the station and meeting the demands for electric of the electric vehicles users at the end of the scheduling cycle.

Charging and discharging power constraint

At any time, the charging and discharging power cannot exceed the limit of the allowed charge and discharge, scilicet : 


$$
\left\{\begin{array}{l}
P c_{\min } \leq P(t) \leq P c_{\max }, P(t)<0 \\
P d_{\min } \leq P(t) \leq P d_{\text {max }}, P(t) \geq 0
\end{array}\right.
$$

where $P c_{\min }$ and $P c_{\max }$ are the minimum and maximum charging power of the centralized charging station; $P d_{\min }$ and $P d_{\max }$ are the minimum and maximum discharging power of the centralized charging station.

Battery power constraint in station

To prevent battery overcharge and over discharge, the power of battery need to be limited. At any time, the critical value of the energy storage of the battery pack cannot be exceeded, scilicet:

$$
C_{\min } \leq C(t) \leq C_{\max }
$$

where $C(t)$ is the state of charge of the battery pack at time $t ; \mathrm{C}_{\min }$ and $\mathrm{C}_{\max }$ are the minimum and maximum value of the state of charge of the battery pack. In this paper, $\mathrm{C}_{\min }$ is 0.1 and $\mathrm{C}_{\max }$ is 0.9 .

User demand constraint

To meet to meet user's demand for electricity, the electricity of battery pack needs to be limited at the end of the scheduling period, scilicet:

$$
\begin{gathered}
C(24) \geq C_{d} \\
C_{d}=E_{d} / E_{c a p} \\
E_{d}=E_{\text {rate }} * N_{d}
\end{gathered}
$$

Where $\mathrm{C}(24)$ is the state of charge of battery pack at the end of scheduling in one day; $C_{d}$ is the required charge state for users in second day; ${ }_{E_{d}}$ is the required electricity for users in second day; $N_{d}$ is that the required number of batteries for user in second day; $E_{\text {rate }}$ is the rated capacity of single battery, whose unit is MWh.

\section{Algorithm implementation}

Day-ahead Scheduling proposed the objective function using PSO, the penalty function method is used to deal with the constraint condition. Take charge and discharge power at each period of the charging station as the position coordinate of the particle, and population can be expressed as $\mathrm{S}=\left\lfloor P_{1,1}, P_{1,2}, \ldots, P_{1,24}, \ldots, P_{n, 1}, P_{n, 2}, \ldots, P_{n, 24}\right\rfloor$.

The PSO of containing penalty function constraint treatment uses the PSO algorithm as a framework. In the iterative process, the evaluation function is constructed by the penalty method. The constrained optimization problem is transformed into an unconstrained optimization problem to deal with. The evaluation function is constructed:

$$
F(x)=f(x)+\lambda(t) H(x)
$$

where $f(x)$ is the initial objective function of the constrained optimization problem; $\lambda(\mathrm{t})=\sqrt{t}$ or $\lambda(\mathrm{t})=t \sqrt{t}$ is the factor of penalty function; $t$ is the number of iterations of PSO algorithm and $H(x)$ is the penalty term. After particle swarm in each update speed and positional information, to judge whether it meets the constraints. If it not satisfied, then increasing the penalty term, the farther away from the constraint conditions, the greater the punishment. If it satisfied, the penalty term is zero. Day-ahead Scheduling the objective function is the minimum value of striking power fluctuations, so the penalty term is always positive.

\section{The strategy of real-time scheduling}

The control objective of Real-time Scheduling is based on the results of Day-ahead Scheduling, smoothing the real-time wind power to the level of average wind power. $1 \mathrm{~min}$ as the scheduling period, making one day divided into 24 periods. The second stage of the power scheduling reference value is shown as (9).

$$
P_{r e f}(t)=P_{w a}(t)-P_{w}(t)
$$

where $P_{r e f}(t)$ is the power scheduling reference at time $t . P_{r e f}(t)>0$ and $P_{r e f}(t)<0$ represents discharge 
and charge respectively. $P_{w a}(t)$ is average wind power and real-time wind power at time $t$.

\section{The operating conditions index of charging station}

To better control the range of battery pack SOC, the reference value of power scheduling should be modified in real time. Therefore, two indexes for evaluating the operating state of the centralized charging station are proposed [13].

1) Battery charge and discharge capacity index

This index is used to measure the residual power of battery pack in centralized charging station, scilicet:

$$
H(t)=\frac{C(t)-C_{r e f}}{\left(C_{\max }-C_{\min }\right) / 2}
$$

where $C_{r e f}$ is the SOC maintenance value of the battery pack, that is, battery pack will locate the vicinity of $C_{r e f}$ after scheduling. The range of $H(t)$ is changing with $C_{r e f} . C(t)$ is closer to the maximum value and it shown that the battery pack energy is more adequate, and discharge capacity is stronger, and discharge index is good, but the ability to charge is very low. $C(t)$ is close to the minimum value and it shown that the battery pack energy is insufficient, and charge capacity is stronger, charge index is good, but the ability to discharge is very low. $C(t)$ is close to $C_{r e f}$, and it shown that the battery pack charge discharge capacity is moderate in current SOC maintenance value.

2) Battery pack charge discharge strength index

This index is used to measure the strength of battery pack to emit or absorb electrical energy, scilicet:

$$
\delta(t)=\omega_{c} \frac{P_{r e f}(t)}{\left|P c_{\max }\right|}+\omega_{d} \frac{P_{r e f}(t)}{\left|P d_{\max }\right|}
$$

where $P_{\text {ref }}(t)$ is the reference value of charging and discharging power. $\delta \in[-1,1] . \delta$ uses -1 to represent that the energy storage power instruction charges to energy storage battery as the maximum charge power. $\delta$ uses 1 to represent that the energy storage power instruction is the energy storage battery discharging to grid as maximum discharging power. And it uses 0 to represent that power instruction reference value is 0 .

\section{Power correction principle}

The overall power scheduling value is the superposition of the power scheduling reference value and the power correction value at second stage, scilicet:

$$
P_{t}(t)=P_{r e f}(t)+f(H, \delta, t)
$$

where $P_{t}(t)$ is the total value of power scheduling value and $f(H, \delta, t)$ is the power correction value at second stage. It has the certain functional relation with two mentioned evaluation indexes, and certainty rules are as follows:

When the SOC is larger, indicating that the battery energy storage is close to saturation. At this moment, if the power reference index $P_{r e f}(t)<0$, charging power should be reduced, that is, correcting $P_{r e f}(t)$ to make it increase, the bigger SOC value is, the bigger correction volume is. If $P_{r e f}(t)>0$, the original value will kept unchanged.

When the SOC is smaller, indicating that the battery energy storage is in sufficient. At this moment, if the power reference index $P_{r e}(t)>0$, the discharging power should be reduced, that is, correcting $P_{r e f}(t)$ to make it decrease, the smaller SOC value is, the bigger the correction volume is. If $P_{r e f}(t)<0$, the original value will kept unchanged.

When SOC is in the vicinity of $C_{r e f}$, the original power index is kept unchanged.

\section{The strategy of power correction}

Concerned the uncertain relationship between power correction value and battery pack, in this 
paper, the fuzzy algorithm is used to solve the power correction value in the real-time scheduling.

The fuzzy input variables are the index of battery pack charging and discharging capability $\mathrm{H}$ and strength index $\delta$. Fuzzy sets are $\{$ NB,NS,ZE,PS,PB $\}, \delta$ 's discourse domain is continuous domain[-1,1], it belongs to membership function shown in Fig.2. Because the index $\mathrm{H}$ domain changes with the maintenance value of SOC, so the variable universe is used. SOC maintenance value is the SOC value in each time after finishing scheduling at first stage, the range of $\mathrm{H}$ domain in each hour is $\left[\alpha_{1}, \alpha_{2}\right]$, where in, $\alpha_{1}=2 *\left(C_{\min }-D(t)\right) /\left(C_{\max }-C_{\min }\right), \alpha_{2}=2^{*}\left(C_{\max }-D(t)\right) /\left(C_{\max }-C_{\min }\right), \mathrm{D}(t)$ is SOC value that $\mathrm{T}$ is located at the end of the hour after finishing scheduling at first stage. $\mathrm{H}$ is belongs to degree function and uses triangular and trapezoidal functions that is similar to $\delta$. The upper and lower bounds of the domain is $\alpha_{1}, \alpha_{2}$, the key turning points are shared equally among the range of the domain.

The output of fuzzy control is the power correction coefficient of battery pack $\Delta F(t)$, and the fuzzy set is $\{\mathrm{NB}, \mathrm{NM}, \mathrm{NS}, \mathrm{ZO}, \mathrm{PS}, \mathrm{PM}, \mathrm{PB}\}$, and the domain of discourse is discrete as $\{-1.0,-0.8,-0.5,0,0.1,0.5,1\}$.

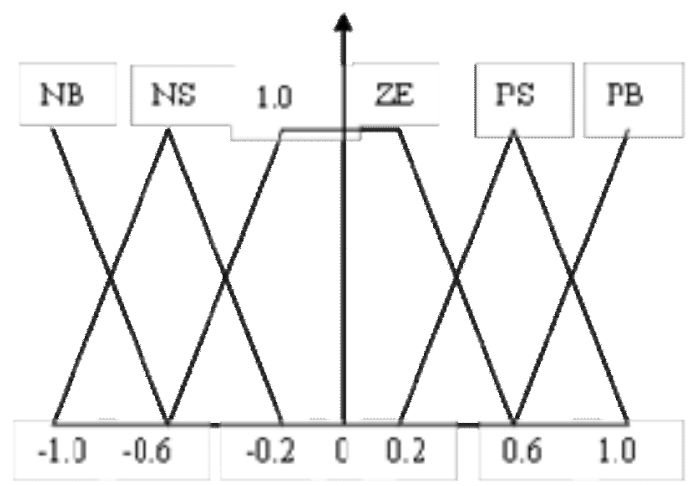

Fig. 2 Charging and discharging intensity membership function

Center of gravity method is used to de-fuzzy, and power correction factor can be obtained at any time.

$$
F(t)=\frac{\sum_{i} \sum_{j} \mu_{H i}\left(H(t-1) \mu_{\delta j}(\delta(t)) \Delta F_{i j}\right.}{\sum_{i} \sum_{j} \mu_{H i}\left(R(t-1) \mu_{\delta j}(\delta(t))\right.}
$$

Battery power command deviation is as follow:

$$
f(H, \delta, t)= \begin{cases}F(t) \times P d_{\text {max }} & F(t)>0 \\ F(t) \times P c_{\text {max }} & F(t) \leq 0\end{cases}
$$

Fuzzy control rule is shown as Table 1.

Table 1. Fuzzy control rule

\begin{tabular}{cccccc}
\hline \multirow{2}{*}{$H$} & \multicolumn{5}{c}{$\delta$} \\
\cline { 2 - 6 } & NB & NS & ZE & PS & PB \\
\hline NB & ZO & ZO & ZO & NS & NB \\
NS & ZO & ZO & ZO & NS & NM \\
ZE & ZO & ZO & ZO & ZO & ZO \\
PS & PM & PS & ZO & ZO & ZO \\
PB & PB & PM & ZO & ZO & ZO \\
\hline
\end{tabular}

\section{Case studies}

A 24-hour typical load curve, which is shown in Table.2.

Two charging devices, 5.8MW for each one, are considered in a centralized station, based on which the rated charging capacity is $11.6 \mathrm{MW}$. The rated capacity of each battery in EV is $17.6 \mathrm{kWh}$, and the rated power of charging and discharging is $3.6 \mathrm{~kW}$. The allowable depth of charging and discharging is 
$10 \%$ to $90 \%$. The initial state of charge is $50 \%$. The energy conversion efficiency of charging and discharging is $90 \%$. The requirement of EV battery for next day is 4500 , and the margin of centralized station is $50 \%$, so the battery number which can be dispatched is 6000 .

Table 2. Daily load data

\begin{tabular}{cc|cc|cc}
\hline $\begin{array}{c}\text { Peri } \\
\text { od }\end{array}$ & $\begin{array}{c}\text { Load/M } \\
\mathrm{W}\end{array}$ & $\begin{array}{c}\text { Perio } \\
\mathrm{d}\end{array}$ & $\begin{array}{c}\text { Load/M } \\
\mathrm{W}\end{array}$ & $\begin{array}{c}\text { Perio } \\
\mathrm{d}\end{array}$ & $\begin{array}{c}\text { Load/M } \\
\mathrm{W}\end{array}$ \\
\hline 1 & 120.72 & 9 & 164.04 & 17 & 165.85 \\
2 & 126.78 & 10 & 156.52 & 18 & 159.35 \\
3 & 133.29 & 11 & 166.23 & 19 & 151.39 \\
4 & 140.45 & 12 & 174.30 & 20 & 144.93 \\
5 & 147.40 & 13 & 181.01 & 21 & 138.65 \\
6 & 155.65 & 14 & 186.94 & 22 & 132.37 \\
7 & 163.47 & 15 & 178.58 & 23 & 126.01 \\
8 & 171.15 & 16 & 171.36 & 24 & 120.80 \\
\hline
\end{tabular}

\section{Analysis of equivalent load curve in day-ahead}

The day-ahead curve of equivalent load and its SOC state in different periods are shown in Fig.3.

The total fluctuation of equivalent power in an hour is about 1015.4MW before the mitigation, which decreases about $92.8 \%$ to $72.7403 \mathrm{MW}$ after the proposed first stage dispatching strategy.

\section{Analysis of refined power in real time}

The dispatching result in real time is shown in Fig.4, the power output of EVs is changing with power reference value and current state of charging. The power dispatching reference is $-0.27083 \mathrm{MW}$ at 23:20, when the state of charging is 0.825248 , which is close to full energy. The charging power is refined to $2.549323 \mathrm{MW}$, which is about $59.7 \%$ of former charging power.
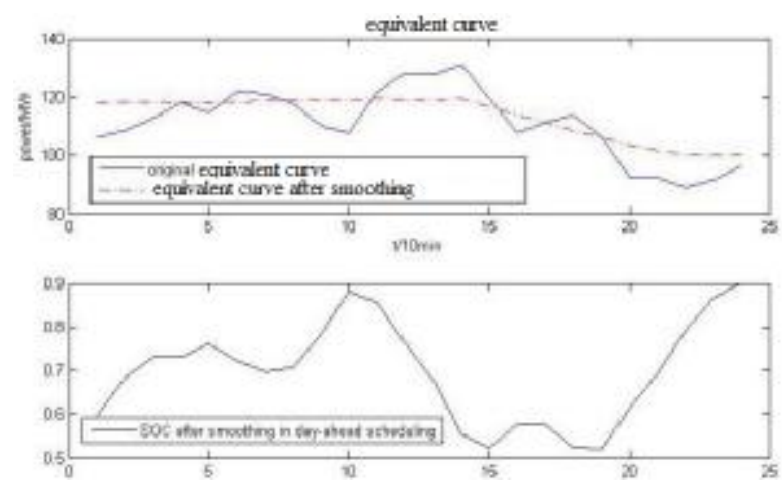

Fig.3 Comparisons of equivalent load curve after day-ahead scheduling
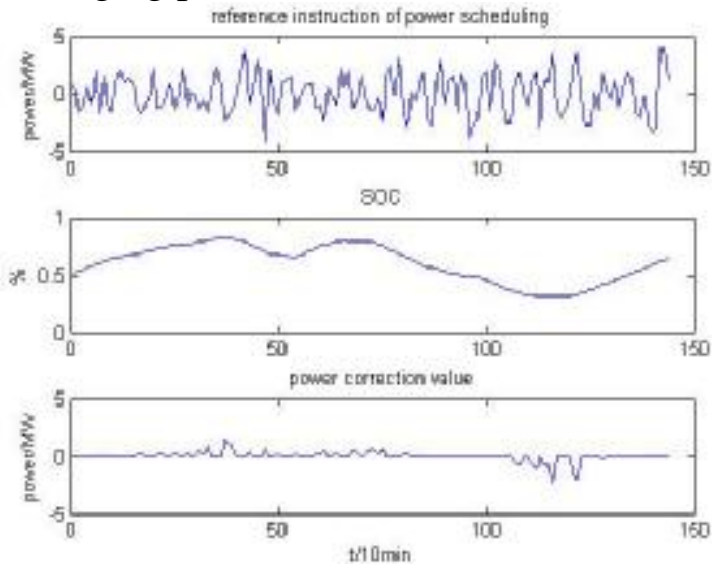

Fig.4 Results of power regulator

\section{Analysis of equivalent load curve in real time}

The total fluctuation of wind power in first $10 \mathrm{mins}$ is about $2901.6 \mathrm{MW}$ before the mitigation, which decreases about $26.3 \%$ to $766.012 \mathrm{MW}$ after the proposed real time dispatching strategy, as shown in Fig.5.

\section{Analysis of equivalent load curve after two-stage dispatching}

The equivalent load curves, which is before and after dispatching respectively, are shown in Fig.6. The charging requirement of EVs can be satisfied and the fluctuation of equivalent load curve is mitigated after the real time scheduling. The total fluctuation of equivalent power in the first 10mins is about $3653.5 \mathrm{MW}$ before the mitigation, which decreases about $12.7 \%$, to $465.4315 \mathrm{MW}$ after the proposed dispatching strategy. 


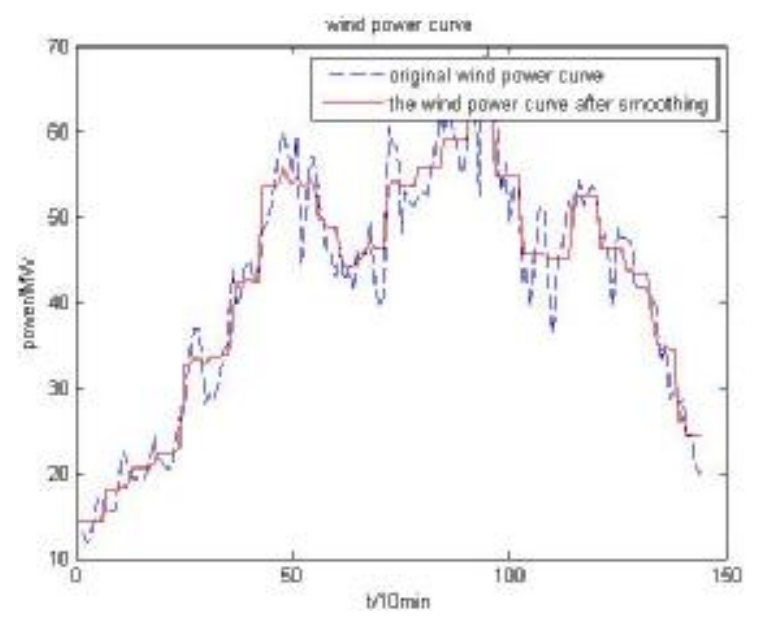

Fig.5 Comparisons of wind power curve before and after smoothing
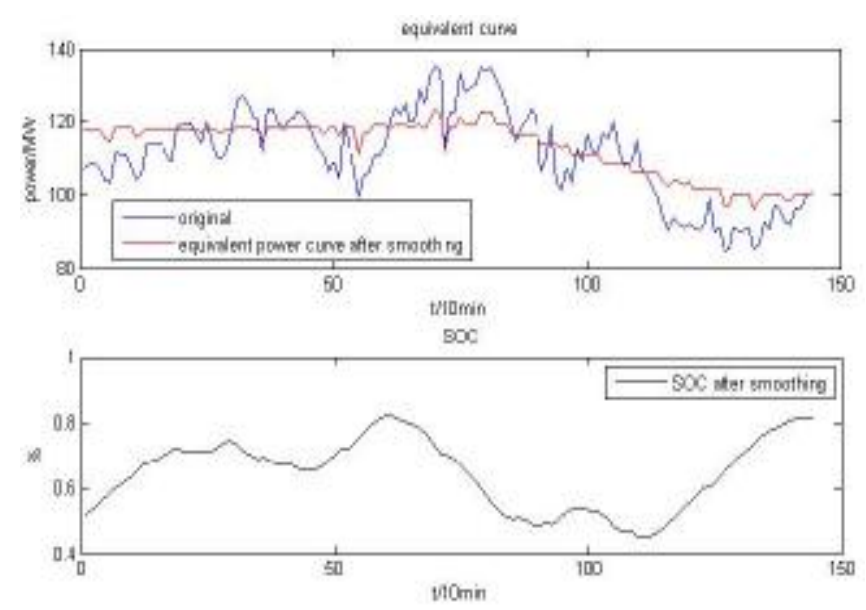

Fig.6 Comparisons of equivalent load curve after two-stage scheduling

\section{Analysis of SOC after the two stage dispatching}

The day-ahead dispatching scheme is obtained, and the SOC is calculated in the first stage dispatching. The results of SOC in day-ahead dispatching are set as the reference values in real time, in order to keep the SOC close to the day-ahead scheme. The SOC values of EVs after dispatching, and the refined power are shown in Fig.7.

\section{Sensitivity analysis of initial SOC}

The initial values of SOC will affect EVs to mitigate the fluctuations of loads. The power fluctuations under different SOC initial values are shown in Fig.8.
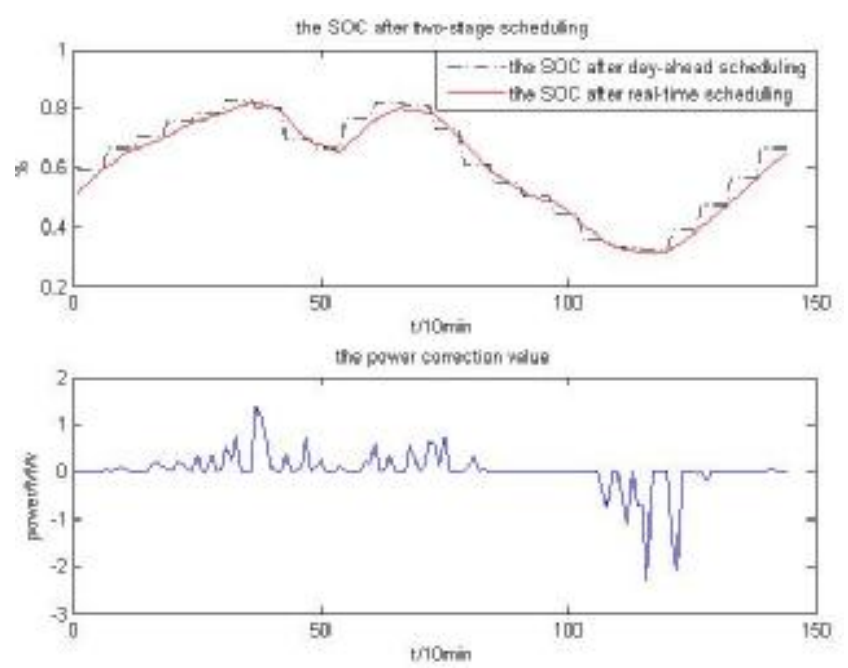

Fig.7 Results of SOC and current power after two-stage scheduling
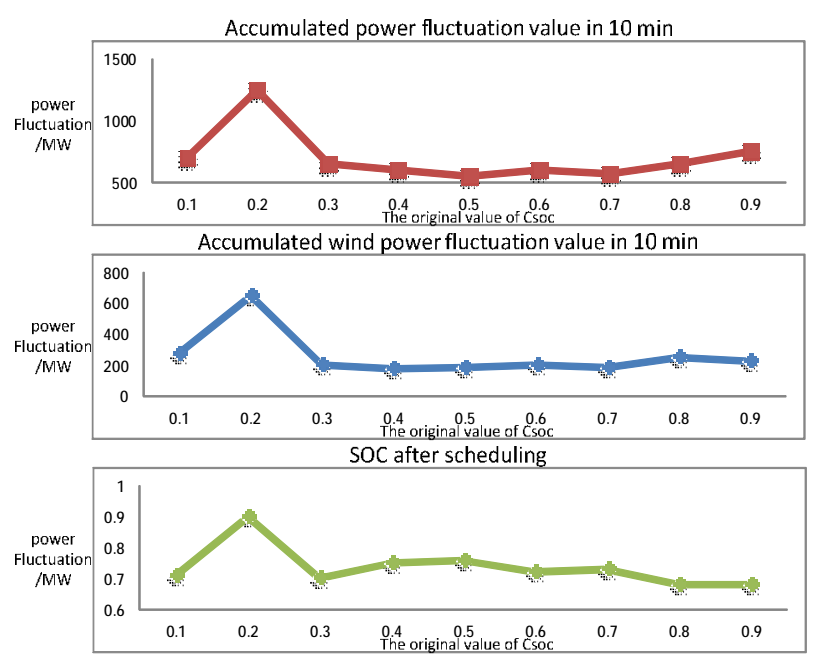

Fig.8 The wind power and equivalent power fluctuation after dispatch of different initial values of SOC

The fluctuation of equivalent curve can be mitigated well when the initial value of SOC is between 0.4 to 0.7 . That is because EV batteries can be charged or discharged flexibly when SOC is near 0.5. On the other hand, the SOC value at the final time, which is always about 0.7 , is not strongly influenced by the initial value, so batteries can be charged and discharged freely and sustainably.

\section{Conclusions}

This paper proposed a two-stage dispatching method for EV centralized station, based on which the requirement of $\mathrm{EV}$ charging is satisfied, and the fluctuation of equivalent power is mitigated. 
The day-ahead dispatching method proposed in this paper can mitigate the fluctuation hourly. Real time dispatching can adjust powers in ten minutes, because the equivalent power curve in an hour fluctuates, especially for wind power.

SOC of EVs may use up or exceed when wind power fluctuates strongly if real time dispatching is not considered.

\section{References}

[1] L. Zhuowei, H. Zechun, S. Yonghua, et al. Coordinated charging and discharging of large-scale plug-in electric vehicles with cost and capacity benefit analysis[J]. Automation of Electric Power Systems, 2012, 36(10): 19-26.

[2] Fernandez L P, Roman T G S, Cossent R. Assessment ofthe impact of plug-in electric vehicles on distributionnetworks[J].IEEE Trans. on Power Systems, 2010, 26(1): 206-213.

[3] Hartmann N, Ozdemir E D. Impact of different utilizationscenarios of electric vehicles on the German grid in2030[J].IEEE Trans. on Power Systems, 2010, 26(1): 206-213.

[4] Y. Xiaolong.Impacts assessment of PHEV charge profiles ongeneration expansion using national energy modeling system[C] //Proceedings of 2008 IEEE Power and Energy SocietyGeneral Meeting: Conversion and Delivery of Electrica1 Energyin the 21st Century, July 20-24, 2008, Pittsburgh, PA, USA: $1-5$.

[5] Clement K, Haesen E, Driesen J. The impact of charging plug - in hybrid electric vehicles on a residential distribution grid[J].IEEE Trans on Power Systems, 2010, 25(1): 371-380.

[6] L. Huiling, B. Xiao-min.Impacts of electric vehicles charging on distribution grid[J]. Automation of Electric Power Systems, 2011, 35(17): 38-43.

[7] B. Yan, J. Jiuchun, Z. Weige, et al.Model and controlstrategy of electric vehicle mobile energy storage system[J].Automation of Electric Power Systems, 2012, 36(22): 36-43.

[8] S. Yonghua, Y. Xia, LU Zongxiang.Integration of plug - in hybrid and electric vehicles: experience from China[c]//Proceedings of Power\& Engineering Society General Meeting. July 25-29, 2010, M inneapolis, M innesota, U SA : 1-5.

[9] G. Ciwei, Z. Liang, X. Fei, et al.Grid planning consideringcapacity and site of large-scale centralized charging stations[J].Proceeding of the CSEE, 2012, 32(7): 40-46.

[10]Xiao H, Ge X, Zhao Z. Day-ahead active distribution network optimal schedule with EV penetration $[\mathrm{C}] / /$ International Conference on Electric Utility Deregulation and Restructuring and Power Technologies. IEEE, 2015.

[11] H. Qilong, J. Qingshan, G. Xiaohong. EV charging load scheduling with high wind power penetration: A robust shortest path approach[C]// 2015:2765-2770.

[12] H. Qilong, J. Qingshan, Q. Zhifeng, et al. Matching EV Charging Load With Uncertain Wind: A Simulation-Based Policy Improvement Approach[J]. IEEE Transactions on Smart Grid, 2015, 6(3):1425-1433.

[13]W. Xifan, S. Chengcheng, W. Xiuli, et al.Survey of electricvehicle charging load and dispatch control strategies[J].Proceedingsof the CSEE, 2013, 33(1): 1-10. 